\title{
Low-cost Thick-film Sensor Based on Piezoelectric Effect for Ballistic Application
}

\author{
Damiano Crescini \\ Dipartimento di Ingegneria dell'Informazione (DII) \\ Università di Brescia, via Branze 38 - 25123, Brescia (Italy)
}

damiano.crescini@ing.unibs.it

\begin{abstract}
:
A new type of pressure sensor is presented which is based on the piezoelectric effect principle in screen printed and fired thick-films. The sensitive element, consisting of a circular piezoelectric layer sandwiched in two conductive layers, is screen printed and fired over an alumina substrate, $2 \mathrm{~mm}$ thickness and $3 \mathrm{~mm}$ diameter. The sensitive element has a structure of a plane capacitor with the piezoelectric film as dielectric and the two conductive layers, based on Pt/Au material, as armatures. Device was incapsulated in a stainless steel housing for the dynamic test. The measuring range covers pressure up to $100 \mathrm{MPa}$ while the reaction time is less than $10 \mu \mathrm{s}$. The experimental analysis shows, in a measuring range up to $100 \mathrm{MPa}$, a sensitivity of about $80-110 \mathrm{pC} / \mathrm{MPa}$. The linearity in the range $25-60 \mathrm{MPa}$ is $\leq 2 \% \mathrm{FSO}$ while in the range $60-150 \mathrm{MPa}$ is $\leq 3 \% \mathrm{FSO}$.
\end{abstract}

Key words: thick-film technology, pressure sensors, piezoelectric sensors, thick-film inks.

\section{INTRODUCTION}

Piezoelectric pressure sensors offer advantages of high sensitivity, good linearity, low hysteresis and wide versatility. Their main use is in the higher cost, precision areas. Lowcost, high volume applications are generally disregarded. Thick-film technology (TFT) is robust, compact and inexpensive. Although commercial thick-film sensors are available, the number of applications has been limited by the lack of specific sensor pastes within the market place. A desirable exercise is to try to merge the advantages of piezoelectric pressure sensors together with thick-film technology to provide a powerful and economic strategy for future sensor development.

Brignell et al. [1] noted that there were three main areas to which thick-film technology could contribute to future sensor development: Firstly, the provision of associated electronic circuitry which can be mounted within the sensor housing. Secondly, the technology allows the creation of support structures, such as electrode patterns, upon which sensing materials can be deposited. The final area concerns the use of the thick-film material itself as the primary sensing element. This paper describes a sensor utilizing all of the above aspects. The primary sensor material is a screen printable thick-film piezoelectric paste, similar to that reported by Baudry in 1987 [2] and Morten et al. in 1991 [3]. It comprises a lead zirconate titanate (PZT) powder, a lead borosilicate glass frit and an organic carrier which serves to give the paste the required viscosity for screen printing. The processing can be carried out using conventional thick-film equipment. Fundamental material studies on the thick-film piezoceramic have revealed that it possesses similar properties to the bulk PZT, the exception being a reduction in the value of the relative permittivity. This is thought to arise 
as a result of the morphology of the film being different to that of the bulk.

\section{DESCRIPTION OF THE STRUCTURE AND MANUFACTURING PROCESS IN THICK- FILM TECHNOLOGY ON CERAMIC}

In order to generate an electrical output from a pressure input, that pressure must first be converted into a proportional displacement or strain. This strain is then transmitted to an electrical transduction element which generates the required signal. Thus, most transducers are comprised of two main components, one mechanical and one electrical. Here a pressure transducers is presented where the mechanical element is the diaphragm, and the electrical elements are the piezoelectric films. This paper will deal with thick-film piezoelectric pressure transducers.

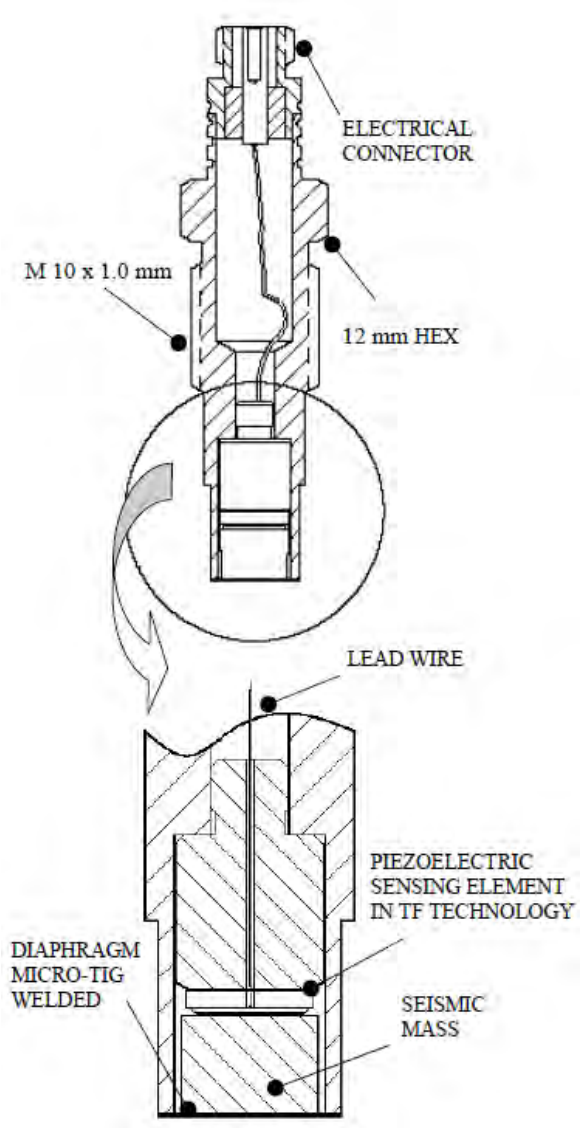

Fig. 1. Cross-section of the TFT pressure sensor

The pressure transducers consist of three basic parts: the transducer housing, the sensing element realized in TFT and the diaphragm for transferring the pressure to the element. Figure 1 illustrates the cross-section of the TFT pressure sensor. The sensitive element, consisting of a circular piezoelectric layer sandwiched in two conductive layers, is screen printed and fired over an alumina substrate, 2 $\mathrm{mm}$ thickness and $3 \mathrm{~mm}$ diameter. The sensitive element has a structure of a plane capacitor with the piezoelectric film as dielectric and the two conductive layers, based on $\mathrm{Pt} / \mathrm{Au}$ material, as armatures [4,5].

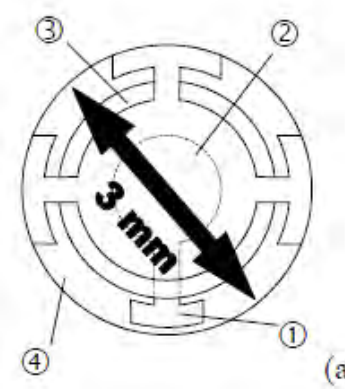

(1) Bottom electrode based on $\mathrm{Pt} / \mathrm{Au}$ ink (2) Top electrode based on $\mathrm{Pt} / \mathrm{Au}$ ink (3) PZT-based piezoelectric film

(4) Ceramic substrate type $\mathrm{Al}_{2} \mathrm{O}_{3} 96 \%$

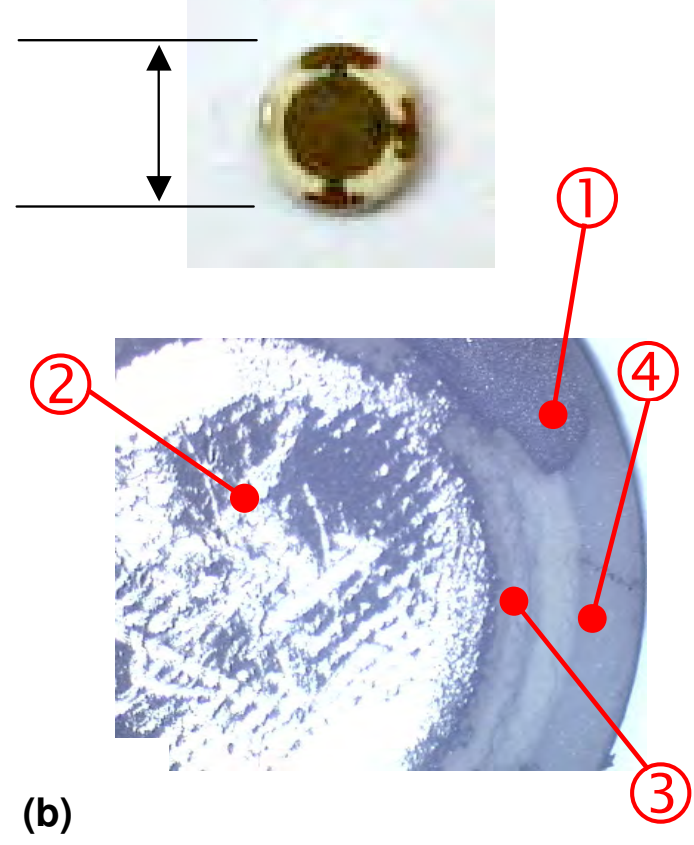

Fig. 2. (a) Schematic view of the TFT sensitive head (b) Photograph of the thick-film sensitive head with enlargement

The piezoelectric film is a PZT ferroelectric layer, $80 \mu \mathrm{m}$ thick; electrical polarization has been obtained by applying, after the firing process, $3 \times 106 \mathrm{~V} / \mathrm{m}$ d.c. electric field for 15 min. at a temperature of $180{ }^{\circ} \mathrm{C}$; after it; without removing the electric field, the material has 
been cooled down to room temperature. In this way, the piezoelectric layer exhibits a $d_{33}$ voltage coefficient of about $180 * 10^{-12} \mathrm{C} / \mathrm{N}$. The device has the polarization direction parallel to the sensitive axis. In figure 2 (a) a sketch of the sensitive head is presented together with the constitutive parts. Figure 2(b) is a photograph of the sensitive head. The circular-shaped alumina substrate, the thick film electrode (gray zone) and the PZT films (white zone) are distinguishable. Figure 2(b) is a photograph of the sensitive head. The circular-shaped alumina substrate, the thick film electrode (gray zone) and the PZT films (white zone) are distinguishable. By means of glassy dielectrics, the top face of the sensing head was bonded to the seismic mass. Figure 3 shows the complete prototype of the piezoelectric pressure sensor in the AISI $316 \mathrm{~L}$ stainless steel housing. Developing high-pressure transducer, the calculation of stress distribution is an important aid in designing the sensitive element. In Figure 4 an example of the Finite Element Analysis is reported. The stress distribution near to the centre of the PZT element is presented due to a pressure load of $600 \mathrm{MPa}$.

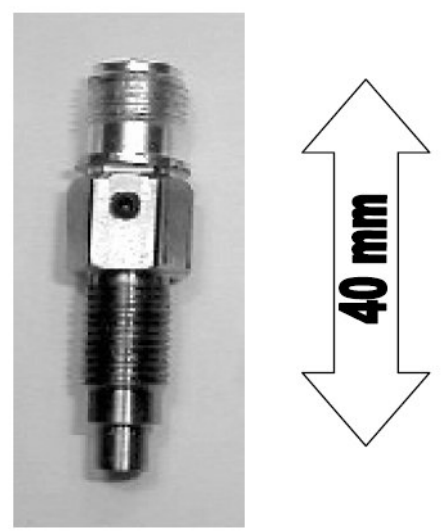

Fig. 3. Photograph of the complete TFT pressure sensor in the AISI 316L housing

\section{EXPERIMENTAL RESULTS}

The TFT pressure sensor has been tested in laboratory in order to evaluate the response of the transducers to known pressures, to verify the dynamic response and to show repeatability. During the testing procedure the following steps have been followed:

- analysis of the linearity and sensitivity;

- $\quad$ analysis of the repeatability;
- analysis of the hysteresis;

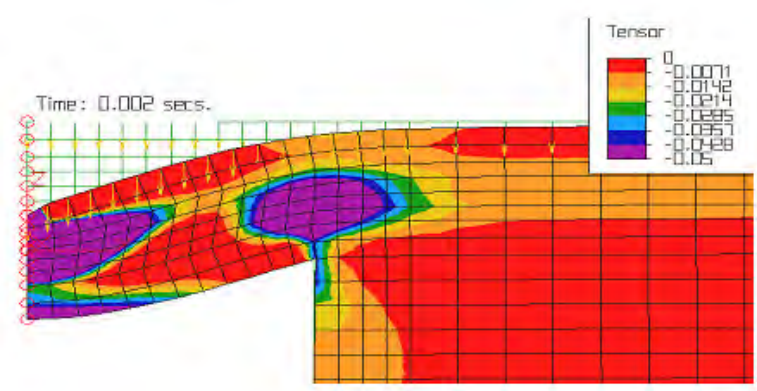

Fig. 4. FEM analysis of the sensitive head

The sensor has been inserted in a dynamic calibrator B620. The calibrator has been used with the aim to determine the following dynamic characteristics of the thick-film sensor:

- dynamic conformity;

- variation of the dynamic characteristic;

- absolute dynamic calibration;

The Model B620 utilizes a rugged, stable, and repeatable AVL quartz 5 QP 2000T-AVL sensor to accurately determine pressure. A free-falling known mass is dropped onto a piston, creating a hydraulic pressure pulse in the pressure calibration cell. The peak pressure can be set in steps of $1 \mathrm{MPa}$ within the range of $25 \mathrm{MPa}$ to $800 \mathrm{MPa}$. The pulse duration is approx. $2.5 \mathrm{~ms}$. The accuracy of the maximum pressure set is 2 $\%$ within the range of 25 to $200 \mathrm{MPa}$ and $1 \%$ within the range of 200 to $800 \mathrm{MPa}$. In figure 5 the sensitivity behavior of the reference pressure sensor is reported. In figure 6 the sensitivity behavior of the tested pressure sensor is reported in the range 25 . 150 MPa. In the course of the curve two zones are individualized: a first zone where the sensitivity changes rapidly with the applied pressure and a second zone where the sensitivity remain constant. This effect is related to the mechanical characteristics of the PZT films. In the measuring range up to $70 \mathrm{MPa}$, a sensitivity from 70 to $120 \mathrm{pC} / 1 \mathrm{Mpa}$ has been found while in the range from 75 to $150 \mathrm{MPa}$ a sensitivity of about $130 \mathrm{pC} / 1 \mathrm{MPa}$ has been found. The linearity in the range $25-60 \mathrm{MPa}$ is $\leq 2 \% \mathrm{FSO}$ while in the range $60-150 \mathrm{MPa}$ is $\leq 3 \% \mathrm{FSO}$. The natural frequency of the TFT prototype is greater than $100 \mathrm{kHz}$ and the rise time is less than $10 \mu \mathrm{s}$. 


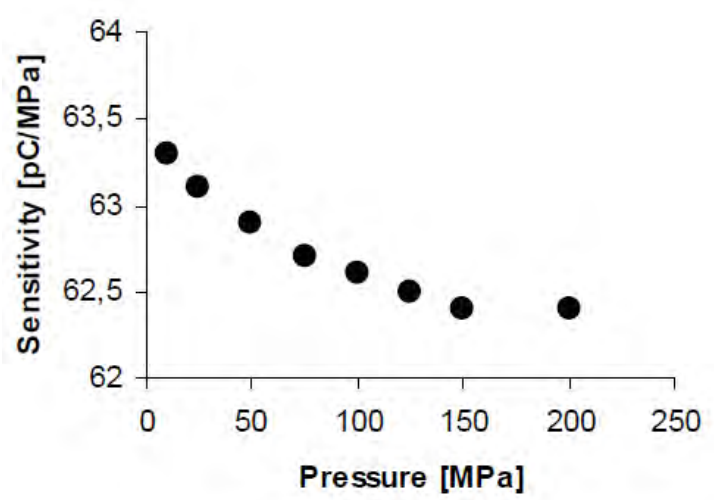

Fig. 5. Sensitivity behaviour of the reference pressure sensor 5 QP 2000T-AVL

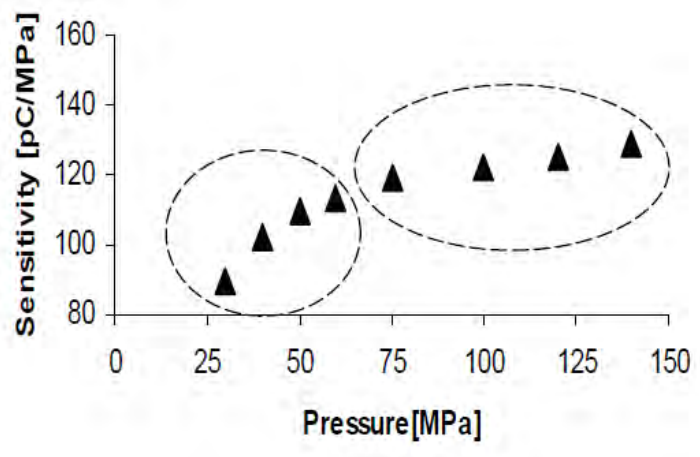

Fig. 6. Sensitivity behavior of the tested TFT pressure sensor in the range up to $150 \mathrm{MPa}$

Test on repeatability and long term stability are still in work. The sensor has been used to evaluate the internal pressure in a gun. The Figure 6 reports the sensor under test, inserted in a cal. 12 hunting gun. In Figure 7 the sensor response is reported in comparison with a commercial high pressure sensor (KISTLER 6203). A good agreement of results is observed.

\section{CONCLUSIONS}

It has been shown in this research that thickfilm ferroelectric/piezoelectric materials with efficient electrical/mechanical conversion can be realized with standard thick-film technology. En example is the pressure sensor described in this paper. The experimental analysis shows in the measuring range up to $70 \mathrm{MPa}$, a sensitivity from 70 to $120 \mathrm{pC} / 1 \mathrm{Mpa}$ while in the range from 75 to $150 \mathrm{MPa}$ a sensitivity of about 130 $\mathrm{pC} / 1 \mathrm{MPa}$ has been found. The linearity in the range $25-60 \mathrm{MPa}$ is $\leq 2 \% \mathrm{FSO}$ while in the range $60-150 \mathrm{MPa}$ is $\leq 3 \% \mathrm{FSO}$. The natural frequency of the TFT prototype is greater than $100 \mathrm{kHz}$ and the rise time is less than 10 $\mu$ s. Future work involves improving piezoelectric and mechanical properties of the thick-film PZT and further analyzing the long term stability and the thermal effect on the sensing head.
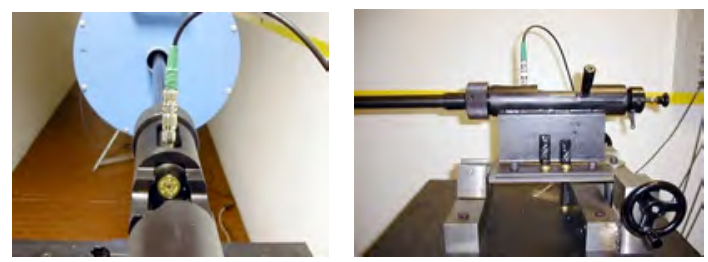

Fig. 7. Photograph of the complete TFT pressure sensor installed in the testing structure (during ballistic measurement)

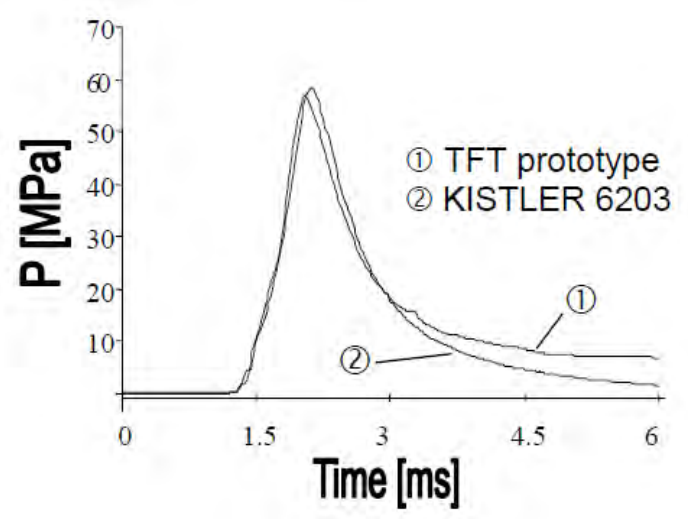

Fig. 8. Dynamic response of the TFT prototype compared with a KISTLER 6203 pressure sensor

\section{References}

[1] Brignell, J. E., White, N. M. and Cranny, A. W. J.:'Sensor applications of thick-film technology', IEE Proc. Pt. I, 1988, 135, No. 4, pp 77-84.

[2] Baudry, H.: 'Screen printing piezoelectric devices', Proc. 6th Eu-rop. Microel. Conf., Bournemouth, 1987, pp 456-463.

[3] Morten, B., De Cicco, G., Gandolfi, A., and Tonelli, C.:'Advances in ferroelectric thick-film materials and sensors', Proc. 8th Europ. Mi-croel. Conf., Rotterdam, 1991, pp 392-399.

[4] D. Crescini, D. Marioli, E. Sardini and A.Taroni .Large bandwith and thermal compensated piezoelectric thick-film accelerometer transducer ., Sensor and Transducer A 87 (2001) pp. 131-138

[5] D. Crescini, D. Marioli, V. Ferrari, and A.Taroni .Vibration and vibratine sensor in thick-film technology., Machine Vibration 4 (1995) pp.161-167 